\title{
Ultrastrukturelle Untersuchungen zur Morphologie und Genese der Spermien von Archaeogastropoda*
}

\author{
R. Kohnert ${ }^{1}$ \& V. Storch ${ }^{2}$ \\ ${ }^{1}$ Zoologisches Institut der Universität; Olshausenstr, 40-60, D-2300 Kiel, \\ Bundesrepublik Deutschland ** \\ ${ }^{2}$ Zoologisches Institut der Universität; Im Neuenheimer Feld 230, D-6900 Heidelberg, \\ Bundesrepublik Deutschland
}

\begin{abstract}
Ultrastructural investigations on the morphology and genesis of sperms in Archaeogastropoda. The sperm cells of Patella coerulea (Patellacea), Monodonta turbinata, and Gibbula tumida (Trochacea) were investigated by means of transmission electron microscopy. They belong to the primitive type (sensu Franzén) and have more features in common with primitive Bivalvia sperms than with Neritacea. Their head contains an apical acrosome and a roundish nucleus followed by 4 or 5 mitochondria and a centriolar apparatus which consists of two centrioles, one of which bears a flagellum. The sperm cells of Monodonta and Gibbula are very similar to each other and differ mainly in size; Patella exhibits more differences (very small acrosome, subacrosomal space, variable number of spherical mitochondria (origin of spermic dimorphism?). The development of the sperm cells shows no peculiarities.
\end{abstract}

\section{EINLEITUNG}

Innerhalb der Diotocardia kommen nach bisherigen Erkenntnissen zwei Spermientypen vor. Während die sich über innere Befruchtung vermehrenden Neritacea Spermien mit langgestrecktem, rohrförmigem Kern (Theodoxus fluviatilis: Tuzét, 1928, 1930, 1950) und stark abgewandelten Mitochondrien (Theodoxus fluviatilis: Giusti \& Selmi, 1982; Kohnert, 1982; Nerita senegalensis: Garreau de Loubresse, 1971; Neritina communis: Kohnert, 1982) besitzen, sind bei den übrigen Archaeogastropoda mit äußerer Befruchtung Spermien mit kurzem, massivem Kern, kappenförmigem Akrosom und vier bis fünf Mitochondrien verbreitet (Personne \& Anderson, 1970; Thompson, 1973; Lewis et al., 1980; Azevedo, 1981).

Lichtmikroskopischen Untersuchungen zufolge sind die Spermien verschiedener Fissurellidae, Trochidae und Turbinidae einander sehr ähnlich; nur in der Größe differieren sie voneinander (Dan, 1956). Detaillierte elektronenoptische Studien an Haliotis rufescens (Lewis et al., 1980) und Patella lusitanica (Azevedo, 1981) zeigen dagegen, daß zwischen den Spermien von Arten verschiedener Familien innerhalb der Diotocardia größere Unterschiede bestehen können.

* Unterstützt durch die Deutsche Forschungsgemeinschaft (DFG Sto 75/4 ff).

** Derzeitige Anschrift: Abteilung Pathologie; Hospitalstr. 42, D-2300 Kiel, FRG. 
Daten über die Spermien weiterer Archaeogastropoda aus der engeren Verwandtschaft der letztgenannten Arten (Patellacea, Patellidae: Patella coerulea) und von entfernter stehenden Taxa (Trochacea, Trochidae: Monodonta turbinata, Gibbula tumida) sollen der Überprüfung dieser Beobachtung dienen.

\section{MATERIAL UND METHODEN}

Patella coerulea Linné und Monodonta turbinata (Born) wurden in Split/Jugoslawien gesammelt, Gibbula tumida (Montagu) stammte von Helgoland.

Nach der Eröffnung der Schale adulter Tiere wurden Hoden und Vas deferens unter eisgekühltem Glutaraldehyd $(2 \%)$ in 0,2 molarem Cacodylatpuffer ( $\mathrm{pH} 7,4)$, der mit Sucrose auf 900 mosm eingestellt war, präpariert und zwei Stunden lang fixiert. Gründlichem Auswaschen in demselben Puffer und zweistündiger Nachfixierung in $4 \%$ iger $\mathrm{OsO}_{4}$-Lösung in Cacodylatpuffer (mit $\mathrm{NaCl}$ auf 850 mosm eingestellt) folgte nach erneutem Auswaschen mit Puffer die Uberführung in Araldit über die Ethanolreihe und Propylenoxid. Am Reichert-Ultramikrotom hergestellte Semidünnschnitte wurden mit Toluidin-Blau angefärbt und zur Orientierung verwendet. Die anschließend hergestellten Ultradünnschnitte wurden mit Uranylacetat in Methanol und Bleicitrat in Wasser nachkontrastiert und mit dem Zeiss EM 9 S-2 elektronenmikroskopisch untersucht.

\section{BEFUNDE}

\section{Die reifen Spermien}

Damit eine Befruchtung wahrscheinlicher wird, müssen Tiere mit äußerer Befruchtung zum Zeitpunkt der Paarung eine große Anzahl reifer Spermien bereithalten. Im Hoden paarungsbereiter Männchen der hier untersuchten Schneckenarten überwiegen dementsprechend reife Spermien, während Stadien der Spermatogenese seltener zu beobachten sind.

Der Grundbauplan der Spermien von Patella coerulea, Monodonta turbinata und Gibbula tumida entspricht dem Typ, den Franzén (1970) für Tiere mit äußerer Befruchtung fordert: Einem vorn leicht zugespitzten, massiven, drehrunden Kern sitzt breit das Akrosom auf. Hinten umringen kugelige Mitochondrien zwei rechtwinklig zueinanderstehende Centriolen. Das hintere setzt sich in der Geissel fort, die mit ihrem $9 \times 2+2$ Muster keine Besonderheiten aufweist.

Während die beiden Trochaceen-Spermien in erster Linie durch Größenunter-

Abb. 1a: Patella coerulea, Längsschnitt durch Vorderende eines Spermiums; b: $P$. coerulea, Längsschnitt durch reifes Spermium; c: $P$. coerulea, Mittelstück aus 5 Mitochondrien, quer; d: Monodonta turbinata, Längsschnitt durch die Basalkörperregion. Beachte die Verdichtungen am distalen Centriol und am Plasmalemm (Pfeile); e: Patella coerulea, Querschnitt durch Mittelstück aus 4 Mitochondrien. Der Kern wurde einmal dicht unterhalb des Akrosoms (1), zum anderen in der Nähe des Mittelstückes getroffen (2). Die äußere Schicht des Akrosoms wird innen mit Leisten verstärkt (Pfeil); f: $M$. turbinata, Kern quer. g: M. turbinata, Akrosom und Mittelstück quer. Die äuReren Membranen benachbarter Mitochondrien verschmelzen miteinander; $h$ : $M$. turbinata, fast ausgereiftes Spermium aus dem Hoden, längs; i: Gibbula tumida, reifes Spermium. Vergr. a, $d-\dot{I}$ $28500 \times$; b, c $14250 \times$ 

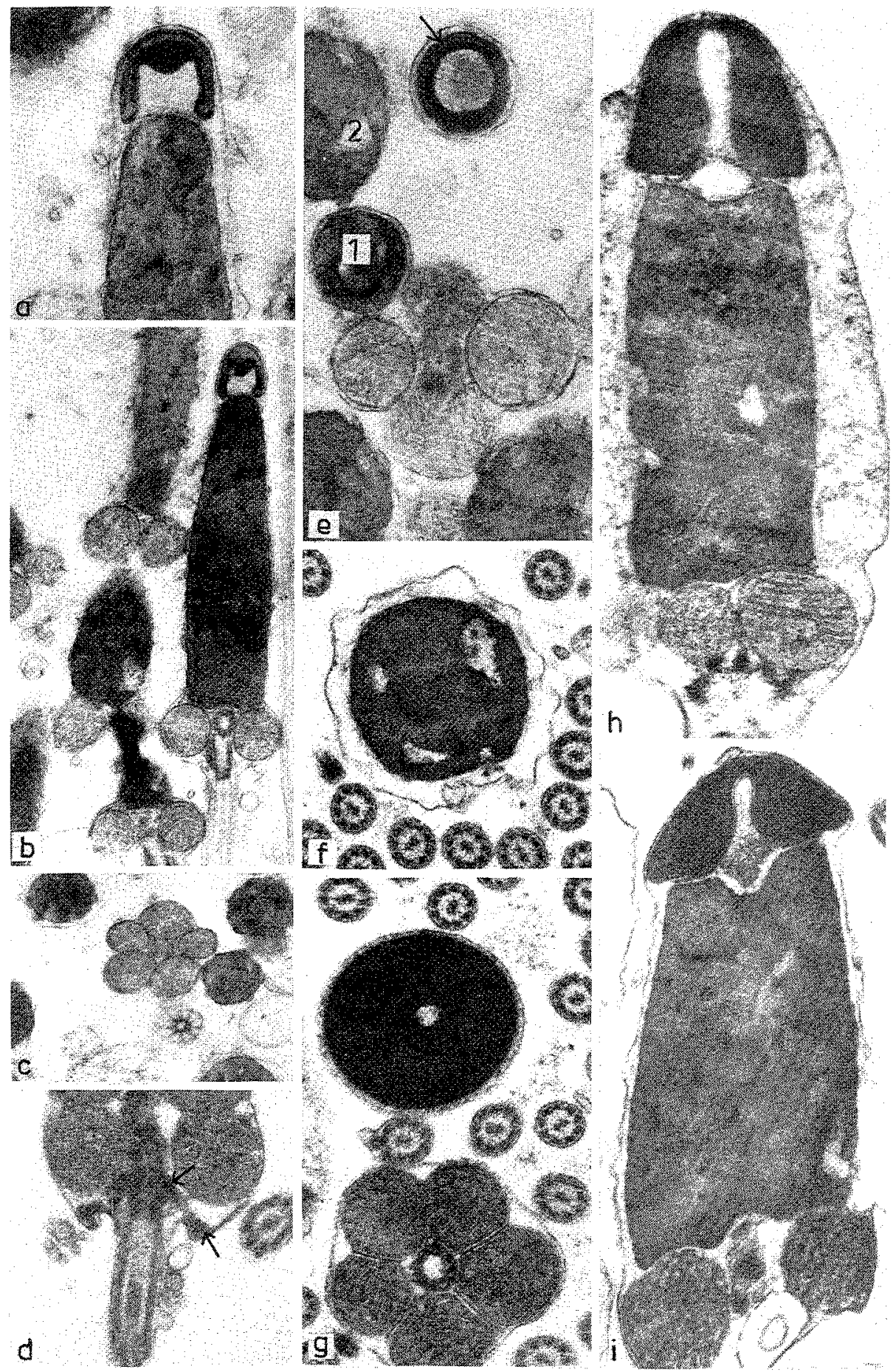
schiede voneinander abgrenzbar sind, lassen sich zwischen diesen und dem Spermium von Patella einige weitere Unterschiede feststellen:

Das recht kleine Akrosom von Patella coerulea mißt in der Länge wie in der Breite lediglich 0,4 $\mu \mathrm{m}$. Wie eine Kappe sitzt es dem unter ihm abgerundeten Kern auf; innen senkt sich sein Dach ein. Die Wandung des etwa 0,2 $\mu \mathrm{m}$ hohen und 0,3 $\mu \mathrm{m}$ durchmessenden Innenraumes ist aus mehreren Schichten aufgebaut, von denen sich drei deutlicher erkennen lassen: Das Innere des Hohlraumes kleidet eine zarte Schicht von Material mittlerer Elektronendichte aus. Sie wird von einer kontrastreichen Wand umfaßt, die seitlich etwa $500 \AA$ stark ist. Apikal, im Bereich der Einsenkung, nimmt ihr Durchmesser auf das Dreifache zu (Abb. 1a, b). Der Bau der äußeren Schicht wird an Querschnitten deutlich: Eine dünne, etwa $80 \mathrm{~nm}$ von der inneren Lage entfernte Wand wird zum Zentrum hin durch Leisten verstärkt, die im Querschnitt kleinen Köpfchen gleichen $(\mathrm{Abb} .1 \mathrm{e})$. Zwischen ihr und den inneren Schichten liegt elektronenoptisch helleres Material. Noch heller sind die zarten Flocken, die den Raum zwischen Akrosom und Kernspitze ausfüllen, so hell, daß dieser auf den ersten Blick leer zu sein scheint (Abb. 1a, b, e).

Sehr viel massiger wirken demgegenüber die Akrosomata von Monodonta turbinata und Gibbula tumida. Auch sie bedecken kappenförmig die Kernspitze, das von Monodonta in Gestalt eines vorn abgerundeten Zylinders mit einer Länge von 0,75 $\mu \mathrm{m}$ und einer Breite von $0,85 \mu \mathrm{m}$ ( $\mathrm{Abb}, 1 \mathrm{~h}$ ), das von Gibbula ist flacher $(0,45 \mu \mathrm{m})$ und breiter $(1,05 \mu \mathrm{m})$ (Abb. 1i). Ein massiver Körper - der Konus - wird vom Kern her im Zentrum von einem etwa $0,1 \mu \mathrm{m}$ weiten Kanal durchbohrt. Apikal erweitert sich der Hohlraum etwas, bevor er dicht unter der Spitze endet. Er ist von einer Membran ausgekleidet, die ein Stück weit in den Spalt zwischen Kern und Akrosom zieht.

Der Abstand zwischen Kern und Akrosom ist bei den Spermien der beiden Trochaceen geringer als bei denen von Patella. Andersartig gebaut ist auch das Kernvorderende: Gegenüber dem in den Konus eingelassenen Hohlraum ist bei beiden Arten die Front zentral eingesenkt. Hier fallen feine Granula auf, die auch den unteren Teil des Kanals ausfüllen (Abb. 1h, i). Kernspitze und Akrosomunterseite verlaufen entlang einer breiten ringförmigen Fläche einander parallel.

Die hinteren Enden der Kerne zeigen im Prinzip den gleichen Bau: Zentral ist eine Mulde ausgebildet, unter der das proximale, schräg oder nahezu senkrecht zur Längsachse gelagerte Centriol ansetzt; weitere kranzförmig angeordnete Mulden überdachen die Mitochondrien des Mittelstückes (Abb. 1i).

Im Mittelstück sind bei Mondonta turbinata und Gibbula tumida regelmäßig fünf Mitochondrien zu beobachten. Die Spermien von. Patella coerulea hingegen besitzen vier oder fünf. Auch in ihrer Anordnung unterscheiden sie sich: bei Patella wird die Kugelform der einzelnen Mitochondrien kaum durch ihre Nachbarn beeinflußt; ein jedes behält seine individuelle Membran (Abb. 1c, e). Die Mitochondrien der Spermien von Monodonta und Gibbula liegen im Gegensatz dazu so eng beieinander, daß benachbarte Mitochondrien abgeflacht werden und ihre Membranen zu deutlich dickeren, radial verlaufenden Wänden verschmelzen. Diese inserieren an den Ecken des von den Mitochondrien gebildeten kurzen fünfseitigen Kanals, der das distale Centriol birgt. Außen enden sie dicht vor dem Plasmalemm (Abb. $1 \mathrm{~g})$.

An der Mitte der Zylinderwand des distalen Centriols setzt bei allen drei Arten eine elektronenoptisch recht dichte amorphe Masse an. Sie verläuft wie ein Kegelmantel 
schräg nach hinten und nimmt dabei an Umfang und Dichte ab. Der Anulus, ein Ring dichteren Materials, der in eine Vorstülpung der Zellmembran eingebettet ist, steht mit ihr in Verbindung (Abb. 1d).

\section{Die Spermiogenese}

Jüngste als Spermatiden ansprechbare Zellen besitzen einen großen Zellkern, dessen Heterochromatin in Form winziger Kömchen mittlerer Elektronendichte homogen wirkt. Es wird aufgelockert durch geräumige, helle und runde Bezirke im Kerninneren sowie durch einen unregelmäßig weiten Saum am Karyolemm. Diese Bereiche durchsetzt flockiges Material von zum Teil großer Elektronendichte. Im Cytoplasma

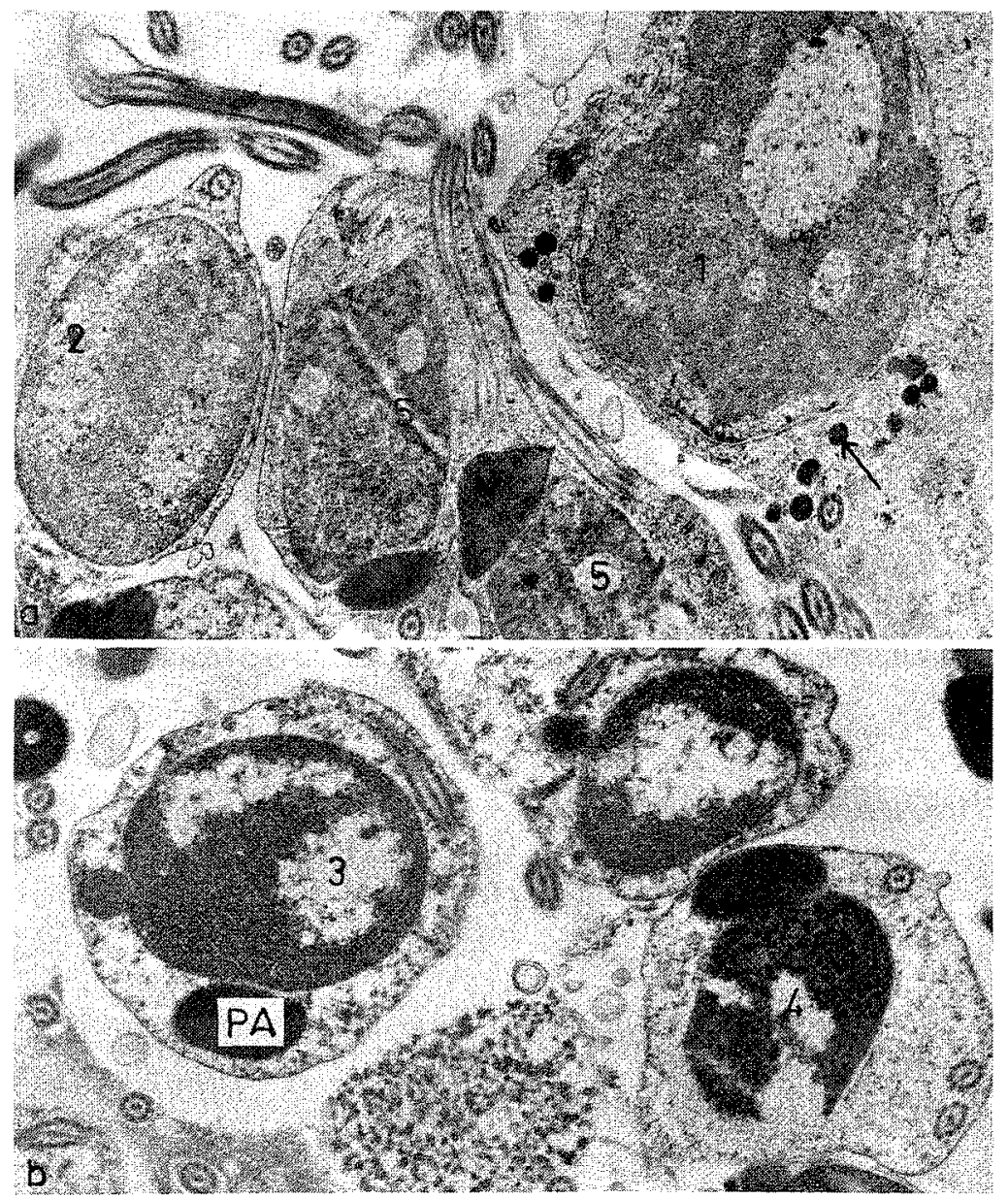

Abb, $2 a_{\text {, }}$ : Monodonta turbinata, Hoden, unterschiedlich weit entwickelte Spermatiden. a: Junge Spermatide (1), in deren Kern bereits die Chromatinkondensation eingesetzt hat. Kugelige Ein* schlüsse hoher Elektronendichte können als Vorläufer des Akrosoms betrachtet werden (Pfeil). 2-5: fortschreitende Chromatinkondensation; b: Proakrosom (PA), das zunächst oval, später pilzhutförmig ist. Beachte die Geisseltubuli im Cytoplasma. Vergr. $14250 \times$ 
fallen in diesem Stadium kugelige Einschlüsse großer Elektronendichte sowie einige weiträumige Zisternen des endoplasmatischen Reticulums auf (Abb. 2a).

Die Chromatinkörnchen wachsen im Laufe der Kernkondensation zu größeren Aggregaten zusammen; gleichzeitig vermindert sich ihre Zahl. Auch die hellen Bereiche bilden sich mehr und mehr zuriick; der Saum am Karyolemm ist in diesem Stadium nicht mehr zu beobachten (Abb. 2b).

Die Entwicklung des Akrosoms geht von den elektronendichten Cytoplasmaeinschlüssen aus. Diese verschmelzen zu einer voluminösen linsenförmigen Masse, die sich dem noch runden Kern eng anlagert (Abb. 2a, b). Zur gleichen Zeit ist bereits die Geissel ausgebildet. Bei jüngeren Stadien liegt sie meist aufgerollt im Cytoplasma (Abb. 2a).

\section{DISKUSSION}

Als einziger Vertreter der Archaeogastropoda, die ein Spermium mit kurzem, massivem Kern besitzen, wurde bislang Patella lusitanica ultrastrukturell untersucht (Azevedo, 1981). Die einzelnen Abschnitte ihrer Spermien sind denen von Patella coerulea sehr ähnlich, nur in den Größenverhältnissen bestehen Unterschiede. Beider Akrosom ist klein und becherförmig, der 'subacrosomale' Raum weit. An Stelle eines Akrosomfilamentes treten spärlich vorhandene Flocken. Die Anzahl der am Aufbau des Mittelstückes beteiligten Mitochondrien ist offensichtlich von Art zu Art unterschiedlich: Azevedo (1981) fand bei Patella lusitanica stets fünf Mitochondrien, vorliegende Untersuchung weist bei Patella coerulea außerdem Mittelstücke mit nur vier Mitochondrien nach. Bei Patella vulgata variiert ihre Anzahl zwischen fünf und sechs (Personne \& Anderson, 1970). Die Mitochondrien aller drei Arten verwachsen nicht miteinander.

Erwähnenswert ist in diesem Zusammenhang eine cytophotometrische Untersuchung von Indelicato \& Streiff (1969) an Patella coerulea: Sie fanden eine zweite, etwa ein Prozent der gesamten Spermien ausmachende Population, die sich als diploid erwies. Es existiert also bereits hier Spermiendimorphismus.

In lichtmikroskopischen Untersuchungen wirken die Spermien verschiedener Überfamilien innerhalb der Archaeogastropoda einander sehr ähnlich, nur in der Größe unterscheidbar (Dan, 1956). Ein Vergleich der bisher untersuchten Patella-Arten mit den in dieser Untersuchung vorgestellten Trochaceen auf ultrastruktureller Basis zeigt indes, daß zumindest zwischen diesen Gruppen charakteristische Unterschiede bestehen. So sind die Akrosomata von Monodonta turbinata und Gibbula tumida mit einem massiven Konus ausgestattet, in den ein enger Kanal eingelassen ist. Ein Akrosomfilament fehlt; wie bei Patella sind statt dessen lose aggregierte Flocken eingelagert. Ebenfalls anders als bei den Patella-Arten ist bei Monodonta turbinata und Gibbula tumida das Mittelstück gebaut. Stets fünf Mitochondrien bilden einen Ring um die beiden Centriolen. Sie sind so eng gepackt, daß ihre benachbarten Membranen sich gegenseitig flach drücken und gemeinsam deutlich dickere Wände bilden. Bei Gibbula cineraria schwankt die Anzahl der Mitochondrien zwischen vier und sechs (Thompson, 1973) bzw. fünf und sechs (Personne \& Anderson, 1970). Bei dieser Art bleiben nach Personne \& Anderson die Mitochondrien unabhängig voneinander.

Patella, Monodonta und Gibbula sind von den Neritacea innerhalb der Archaeogastropoda und, soweit bekannt, von sämtlichen Monotocardia auf Grund mehrerer Merk- 
male abzugrenzen, die teils für eine, teils für beide Gruppen Gültigkeit haben: Es fehlen (1) Akrosomfilament und (2) Akrosomvesikel. (3) Zwei Centriolen im reifen Spermium werden von (4) vier bis fünf (sechs) nicht oder nur geringfügig veränderten Mitochondrien umgeben. (5) Distal vom Anulus wird bei den Patella-Arten und den betrachteten Trochaceen die Geissel nur von einem sehr dünnen Cytoplasmamantel umgeben. Bei den übrigen Prosobranchiern ist dieser Mantel wesentlich stärker und enthält PAS(Tochimoto, 1967) bzw. PA-TSC-SP-positive Substanzen (Anderson \& Personne, 1976). Es handelt sich um Glykogen, das in einer Form vorliegt, die keiner besonderen Nachweismethode bedarf, um im elektronenoptischen Bild sichtbar zu werden. Im Mittelstück der 'primitiven' Spermien gespeichertes $\beta$-Glykogen wird dagegen erst durch Anwendung der PA-TSC-SP-Methode sichtbar (Anderson \& Personne, 1976).

Mehr Gemeinsamkeiten sind demgegenüber mit den Spermien einiger Bivalvier festzustellen: Auch hier sind die Reservestoffe auf die Umgebung der Mitochondrien beschränkt (Anderson \& Personne, 1976). Spermien, die sowohl Patellaceen- als auch Trochaceen-Merkmale tragen, fanden Popham \& Marshall (1977) bei Nucula hartvigiana vor. Deren Akrosom und der schlanke konische Kern ähneln sehr den Verhältnissen bei Patella. Der becherförmige Konus des Akrosoms ist nicht massiv, der Hauptteil des elektronendichten Materials liegt im proximalen, kernfernen Abschnitt. Im 'subacrosomalen' Raum befindet sich wie bei Patella und den Trochaceen flockiges Material. Das Mittelstück erinnert mehr an die Trochaceen: Meist fünf (bisweilen sechs) Mitochondrien sind so eng gepackt wie bei Monodonta turbinata und Gibbula tumida. Ob auch hier die benachbarten äußeren Membranen zu stärkeren Wänden vereint werden, erwähnen die Autoren nicht.

Die Akrosomata verschiedener weiterer Bivalvia (Galtsoff \& Philpott, 1960: Crassostrea virginica; Brandriff et al., 1978: Crassostrea gigas) erinnern in der Form an die Akrosomata der hier vorgestellten Trochaceen. Als wesentlicher Unterschied ist bei diesen Bivalviern die Aufteilung des Konus in mindestens zwei Helligkeitszonen hervorzuheben, ferner die tief in den Kern eingeschnittene 'nuclear fossa', die als Fortsetzung des 'subacrosomal space' bei den Crassostrea-Arten ausgebildet ist. Diese Merkmale sind auch typisch für die Spermien von Haliotis rufescens (Pleurotomariacea, Archaeogastropoda), die von Lewis et al. (1980) beschrieben wurden. Hier liegt, anders als bei den oben aufgeführten Bivalvia, die elektronendichtere Zone des Konus kernfern.

\section{ZITIERTE LITERATUR}

Anderson, W. A. \& Personne, P., 1976. The molluscan spermatozoon: Dynamic aspects of its structure and function. - Amer. Zool. 16, 293-313.

Azevedo, C., 1981. The fine structure of the spermatozoon of Patella lusitanica (Gastropoda: Prosobranchia), with special reference to acrosome formation. - J. submicrosc. Cytol. 13, 47-56.

Brandriff, B., Moy, G. W. \& Vacquier, V. D., 1978. Isolation of sperm bindin from the oyster (Crassostrea gigas). - Gamete Res. 1, 89-100.

Dan, J. C., 1956. The acrosome reaction. - Int. Rev. Cytol. 5, 365-393.

Franzén, A., 1970. Phylogenetic aspects of the morphology of spermatozoa and spermiogenesis. In: Comparative spermatology. Ed. by B. Baccetti. Acad. Pr., New York, 29-46.

Galtsoff, P. S. \& Philpott, D. E., 1960. Ultrastructure of the spermatozoon of the oyster, Crassostrea virginica. - J. Ultrastruct. Res. 3, 241-253.

Garreau de Loubresse, N., 1971. Spermiogenèse d'un gastéropode prosobranche: Nerita senegalensis; évolution du canal intranucléaire. - J. Microsc. 12, 425-440. 
Giusti, F. \& Selmi, M. G., 1982. The morphological peculiarities of the typical spermatozoa of Theodoxus fluviatilis (L.) (Neritoidea) and their implications for motility. - J. Ultrastruct. Res. 78, 166-177.

Indelicato, J. \& Streiff, W., 1969. Etude statistique et cytophotométrique de la dimégalie des spermatozoides chez Patella coerulea L., mollusques, gastêropodes, prosobranches. - C. r. hebd. Séanc. Acad. Sci.., Paris (D) 268, 2091-2094.

Kohnert, R., 1982. Vergleichende ultrastrukturelle Untersuchungen zur Morphologie und Genese eupyrener Spermien der Prosobranchier. Diss., Univ. Kiel, $157 \mathrm{~S}$.

Lewis, C. A., Leighton, D. L. \& Vacquier, V. D., 1980. Morphology of Abalone spermatozoa before and after the acrosome reaction. - J. Ultrastruct. Res. 72, 39-48.

Personne, P. \& Anderson, W., 1970. Etude comparée des évolutions mitochondriales au cours de la spermatogenèse chez quelques gastéropodes. In: Comparative spermatology. Ed. by B. Baccetti. Acad. Pr., New York, 393-400.

Popham, J. D. \& Marshall, B., 1977. The fine structure of the spermatozoon of the protobranch bivalve, Nucula hartvigiana Pfeiffer-Veliger 19, 431-433.

Thompson, T. E., 1973. Euthyneuran and other molluscan spermatozoa. - Malacologia 14, $167-206$.

Tochimoto, T., 1967. Comparative histochemical study on the dimorphic spermatozoa of the Prosobranchia with special reference to polysaccharides. - Sci. Rep. Tokyo Kyoiku Daig (B) 13, 75-109.

Tuzét, O., 1928. Sur la spermatogenèse de Theodoxia fluviatilis (Sand.), Bythinia tentaculata (L.) et Cyclostoma elegans (Müll.), - C. r. Séanc. Soc. Biol. 99, 124-125.

Tuzét, O., 1930, Recherches sur la spermatogenèse des Prosobranches. - Archs Zool. exp. gén. 70, 95-229.

Tuzét, O., 1950. Le spermatozoide dans la série animale. - Revue suisse Zool. 57, 433-451. 